\title{
RESEARCH
}

Open Access

\section{Usefulness of the triglyceride glucose-body mass index in evaluating nonalcoholic fatty liver disease: insights from a general population}

Rongsheng Wang ${ }^{1}$, Longlong DAl ${ }^{2}$, Yanjia Zhong ${ }^{3}$ and Guobo Xie ${ }^{4^{*}}$ (i)

\begin{abstract}
Background: Triglyceride glucose-body mass index (TyG-BMI) is a recently developed alternative indicator to identify insulin resistance. However, few studies have investigated the association between the TyG-BMI and nonalcoholic fatty liver disease (NAFLD). Therefore, this study aimed to study the relationship between NAFLD and the TyG-BMl in the general population and its predictive value.

Methods: A cross-sectional study was conducted on 14,251 general subjects who took part in a comprehensive health examination. The anthropological characteristics and many risk factors for NAFLD were measured.

Results: After fully adjusting for confounding variables, a stable positive correlation was found between NAFLD and the TyG-BMI (OR: 3.90 per SD increase; 95\% Cl: 3.54 to 4.29; P-trend<0.00001). This positive correlation was not simply linear but a stable non-linear correlation. Additionally, obvious threshold effects and saturation effects were found, in which a threshold effect occurred when the TyG-BMI was between 100 and 150; when the TyG-BMI was between 300 and 400, the corresponding NAFLD risk appeared saturated. Furthermore, receiver operating characteristic analysis showed that the TyG-BMI could better predict the risk of NAFLD than other traditional indicators [TyG-BMI (AUC): 0.886; 95\% Cl: 0.8797-0.8927; $P<0.0001]$, particularly among young and middle-aged and non-obese people.

Conclusions: This epidemiological study is the first on the association between the TyG-BMI and NAFLD risk in the general population. In this large data set from the general population, the TyG-BMI showed an independent positive correlation with NAFLD. The discovery of the threshold effect and saturation effect between them provides a new idea to prevent and treat NAFLD.
\end{abstract}

Keywords: Triglyceride, Body mass index, Triglyceride, Triglyceride glucose-body mass index, Triglyceride-glucose index

\footnotetext{
* Correspondence: xgb19810830@163.com

${ }^{4}$ Department of Cardiology, Jiangxi Provincial People's Hospital Affiliated to

Nanchang University, Nanchang, P.R. China 330006

Full list of author information is available at the end of the article
}

(C) The Author(s). 2021 Open Access This article is licensed under a Creative Commons Attribution 4.0 International License, which permits use, sharing, adaptation, distribution and reproduction in any medium or format, as long as you give appropriate credit to the original author(s) and the source, provide a link to the Creative Commons licence, and indicate if changes were made. The images or other third party material in this article are included in the article's Creative Commons licence, unless indicated otherwise in a credit line to the material. If material is not included in the article's Creative Commons licence and your intended use is not permitted by statutory regulation or exceeds the permitted use, you will need to obtain permission directly from the copyright holder. To view a copy of this licence, visit http://creativecommons.org/licenses/by/4.0/ The Creative Commons Public Domain Dedication waiver (http://creativecommons.org/publicdomain/zero/1.0/) applies to the data made available in this article, unless otherwise stated in a credit line to the data. 


\section{Background}

Nonalcoholic fatty liver disease (NAFLD) is the most common and fastest-growing liver disease. Recent epidemiological surveys have estimated the global prevalence of NAFLD at approximately 25\%, which is expected to increase to $33.5 \%$ by 2030 [1-3]. NAFLD may develop into nonalcoholic steatohepatitis, compensated/decompensated cirrhosis, or even hepatocellular carcinoma [1, 4], while extrahepatic NAFLD can aggravate cardiovascular and cerebrovascular diseases, diabetes, and kidney disease with adverse consequences [5-7]. Despite the increasing prevalence of NAFLD and its adverse effects on multiple systems throughout the body, no definite drug treatment currently exists except for lifestyle changes through exercise [8]. Therefore, identifying the population prone to NAFLD early through simple and effective diagnostic tools in daily practice is beneficial.

Insulin resistance (IR) refers to reduced peripheral tissue insulin sensitivity, characterized by impaired glucose uptake and oxidation, and it participates in the key link of the pathogenesis of NAFLD [8, 9]. The triglycerideglucose (TyG) index is a compound index that combines fasting blood glucose (FPG) and fasting triglyceride (TG) and can better reflect IR. Because of its convenient acquisition and simple calculation, it has been widely accepted and used clinically [10-12]. In a recent study, Er et al. found that the combination of the body mass index (BMI) and TyG index can simultaneously reflect the information of many important clinical indexes, such as blood glucose, blood lipids and the BMI, and can better reflect IR than the TyG index alone [13]. Considering the importance of IR in the pathogenesis of NAFLD $[8,9]$, researchers have speculated that the TyG-BMI may be a good marker to predict NAFLD in the general population. The existing several studies of separately considering the non-obese or overweight, obese people [14-16]. However, it remains unclear in which BMI population the TyG-BMI has better NAFLD identification ability, and whether the TyG-BMI and NAFLD are linear or non-linear associations is also an unresolved clinical issue. Therefore, this study aimed to study the association between the TyGBMI and NAFLD in the general population and its predictive value, analyse the relationship between them in detail and the interaction with other factors and provide new ideas to prevent and treat NAFLD.

\section{Methods}

\section{Study design and subjects}

The NAGALA study is an ongoing cohort study, and the design details of the study have been described in previous studies [17]. Briefly, the NAGALA study project recruited 20,944 general subjects who participated in a comprehensive health examination at Murakami Memorial Hospital from 2004 to 2015. The project aimed to assess risk factors for common chronic diseases from health check-up information in the general population.

This study was a post hoc analysis of the public data in the NAGALA cohort, and the original data were uploaded to the DRYAD database by Professor Fukui [18]. To make the data better serve the human population, according to the content of the public data usage protocol of the Dryad database, each researcher can use the public data of the database for secondary analysis.

Based on previous studies, this study established a new hypothesis: in the general population, is TyG-BMI and NAFLD related? According to the purpose of the study, a cross-sectional design was adopted, and the following exclusion criteria were established: 1) subjects diagnosed with viral hepatitis or impaired fasting glucose or diabetes at baseline $(N=1547) ; 2)$ subjects who were taking medication at baseline $(N=2321)$; 3) subjects with incomplete covariable data $(N=873)$; 4$)$ alcohol consumption: male $\geq 210 \mathrm{~g} / \mathrm{w}$ or female $\geq 140 \mathrm{~g} / \mathrm{w}$ [19]. Because Murakami Memorial Hospital's institutional ethics committee had authorized the previous study, separate ethical approval was no longer required for this study [17]. Additionally, because the identification information of the subjects was deleted from the data and uniformly replaced by the health check code and the informed consent for each subject on using the data in the previous study was obtained, the application to obtain informed consent again was not required in this study. The entire study protocol followed the Declaration of Helsinki.

\section{Health check-up and laboratory measurement}

The basic health check-up information of the subjects was recorded by trained medical staff using a standard and unified questionnaire, including height, sex, habit of exercise, systolic/diastolic blood pressure (S/BBP), age, smoking status, waist circumference (WC), weight, and drinking status. Blood samples for biochemical analysis were obtained after an overnight fasting period of at least $8 \mathrm{~h}$. Analysis indicators included alanine aminotransferase (ALT), haemoglobin A1c (HbA1c), TG, gamma-glutamyl transferase (GGT), total cholesterol (TC), aspartate aminotransferase (AST), high-density lipoprotein cholesterol (HDL-C) and FPG.

\section{Definitions and calculations}

$\mathrm{BMI}=$ weight $/$ height $^{2}$. TyG $=\mathrm{Ln}[(\mathrm{FPG}(\mathrm{mg} / \mathrm{dL}) / 2) \times \mathrm{TG}$ $(\mathrm{mg} / \mathrm{dL}) \times]$ [11]. TyG-BMI $=\mathrm{BMI} \times \mathrm{TyG}$ [13]; drinking status: according to the amount of alcohol consumed per week, classified as non or small $(<40 \mathrm{~g} / \mathrm{w})$, light (40-139 g/w) and moderate (140-209 g/w); smoking status: at baseline data access, classified as nons/ former/current smokers based on smoking history; Habit of exercise: Defined as at least once a week to attend a physical exercise. 


\section{Diagnosis of NAFLD by abdominal ultrasonography}

After abdominal ultrasonography was conducted by a trained technician, experienced gastroenterologists examined the sonograms and made a comprehensive judgement and NAFLD diagnosis based on the sonographic features of the four types of deep attenuation, ultrasonic hepatic vascular imaging, ultrasound comparison of the liver and kidney and brightness of the liver under ultrasound without knowing the information of the participants [20].

\section{Statistical analysis}

This study makes a statistical analysis of TyG-BMI as a categorical variable (the quintile of the TyG-BMI was calculated using a quantile function divided into five groups) and a continuous variable to deeply understand the association between NAFLD and the TyG-BMI. The main statistical analysis process was as follows:

Step one (statistical description and analysis of the baseline data): Continuous variables in this study were described as the mean [standard deviation (SD)] or median (interquartile range), and the differences among TyG-BMI groups were evaluated by the Kruskal Wallis $\mathrm{H}$ test and Steel Dwass test or one-way ANOVA and Tukey's HSD test. Categorical variables were described as frequencies (\%), and chi-squared test was used to check the differences between the TyG-BMI groups.

Step two (association analysis of TyG-BMI and NAFL D): Before establishing the multivariable logical regression model, the researchers first checked the collinearity between the covariables (Supplementary Table 1) [21]. Next, the correlation between the baseline data and TyG-BMI was checked by linear regression (Supplementary Table 2) [22]. The variables significantly related to TyG-BMI were considered auxiliary factors of the relationship between the two. These auxiliary factors will be included as important adjustment variables in the multivariate regression model. After the above steps were completed, multiple logistic regression was used to calculate the odds ratio (OR) and 95\% confidence interval (CI) before and after adjustment to further explore the association between NAFLD and TyG-BMI. To further ensure the stability of the data analysis, the researchers further processed the TyG-BMI quintile as a continuous variable to conduct a trend test. In this study, adjustment of the multivariable logical regression model follows the STROBE statement [23] and shows the results according to different adjustment methods and adjustment degrees through several models, in which the crude model does not adjust any covariates; model 1 adjusted for general demographic variables; model 2 adjusted the non-collinear variables in which the impact of TyG-BMI on NAFLD risk was more than $10 \%$ and the non-collinear variable of $P<0.05$ in univariate analysis [24]; model 3 adjusted for noncollinear variables associated with TyG-BMI. Additionally, the research team used the generalized additive model to analyse the smoothing function to simulate the potential non-linear relationship between NAFLD and the TyG-BMI.

Step three (Hierarchical analysis): To verify whether the correlation between the TyG-BMI and NAFLD was different among different populations, the research team conducted exploratory hierarchical analysis using a hierarchical logistic regression model in some subgroups and examined the differences between different stratifications using a likelihood ratio test to determine whether an interaction occurred.

Step four (ROC curve analysis of NAFLD-related indicators): Previous research has shown that the BMI, WC, lipids, the lipid ratio, blood glucose, the TyG index and related parameters of NAFLD have better prediction performance $[16,25,26]$. In this study, the predictive value of WC, the BMI, the TyG-WC, TyG index, TG/ HDL-C ratio, the TyG-BMI and other factors related to NAFLD was analyzed by constructing receiver operating characteristic curve (ROC).

All the statistical analysis processes were based on the $\mathrm{R}$ language-based Empower (version 2.0) statistical software, with a 2 -sided significance threshold of $p<0.05$.

\section{Results}

Basic clinical and laboratory characteristics

In the present study, 14,251 eligible subjects were evaluated. According to the quintile of the TyG-BMI, the subjects were equally divided into 5 groups. The clinical and laboratory characteristics of each TyG-BMI group are recorded in Table 1 . The average age of the subjects was 43.53 years, $52 \%$ of the subjects were male, and the prevalence rate of NAFLD was $17.59 \%$. The subjects with a higher TyG-BMI value had a higher male proportion, an older age, a higher BMI, a higher WC, a higher TyG index, a higher blood pressure, a higher prevalence of NAFLD, a higher proportion of smoking and drinking, and fewer people who maintained exercise habits. Regarding the laboratory parameters, except for HDL-C and TyG-BMI that showed an inverse trend, most of the other laboratory parameters also maintained a trend similar to that of the clinical indicators; among the participants with higher TyG-BMI values, AST, HbA1c, TC, GGT, FPG, ALT, and TG were higher.

\section{Association analysis of NAFLD and the TyG-BMI}

As described in the second step of statistical analysis, the association between NAFLD and the TyG-BMI was mainly analysed in multiple models using the TyG-BMI as a categorical variable and a continuous variable. In the crude model, NAFLD and TyG-BMI was positively 
Table 1 Baseline characteristics of five groups

\begin{tabular}{|c|c|c|c|c|c|c|}
\hline & \multicolumn{5}{|l|}{ TyG-BMI quintile } & \multirow[b]{2}{*}{$P$-value } \\
\hline & Q1(97.48-147.69) & Q2(147.72-164.94) & Q3(164.95-182.05) & Q4(182.07-205.33) & Q5(205.34-421.32) & \\
\hline No. of participants & 2850 & 2850 & 2851 & 2849 & 2851 & \\
\hline Age (years) & $40.18(8.39)$ & $42.98(8.70)$ & $44.39(8.93)^{*}$ & $45.35(8.97)^{*}$ & $44.77(8.50)$ & $<0.001$ \\
\hline Weight (kg) & $48.32(5.59)$ & $54.08(6.16)$ & $59.33(6.80)$ & $65.00(7.04)$ & $74.57(10.04)$ & $<0.001$ \\
\hline Height (cm) & $161.50(7.36)$ & $163.06(8.31)$ & $164.94(8.62)$ & $166.74(8.33)$ & $167.74(8.16)$ & $<0.001$ \\
\hline BMI $\left(\mathrm{kg} / \mathrm{m}^{2}\right)$ & $18.49(1.30)$ & $20.29(1.11)$ & $21.75(1.14)$ & $23.34(1.26)$ & $26.45(2.56)$ & $<0.001$ \\
\hline$W C(\mathrm{~cm})$ & $66.42(4.84)$ & $71.14(4.99)$ & $75.57(5.11)$ & $80.26(5.01)$ & $87.54(6.92)$ & $<0.001$ \\
\hline Sex (male) & $538(18.88 \%)$ & 1046 (36.70\%) & 1548 (54.30\%) & 2034 (71.39\%) & 2245 (78.74\%) & $<0.001$ \\
\hline NAFLD & $11(0.39 \%)$ & $56(1.96 \%)$ & $198(6.94 \%)$ & $612(21.48 \%)$ & 1630 (57.17\%) & $<0.001$ \\
\hline Habit of exercise & $438(15.37 \%)$ & $548(19.23 \%)$ & $547(19.19 \%)$ & $497(17.44 \%)$ & $440(15.43 \%)$ & $<0.001$ \\
\hline ALT (IU/L) & $13(11-17)$ & $14(11-18)$ & $16(12-20)$ & $19(15-25)$ & $25(18-35)$ & $<0.001$ \\
\hline AST (IU/L) & $16(13-19)^{* *}$ & $16(13-19)$ & $16(14-20)$ & $18(14-21)$ & $20.00(16-25)$ & $<0.001$ \\
\hline GGT (IU/L) & $12(10-14)$ & $13(10-16)$ & $14.00(11-19)$ & $17.00(13-24)$ & $22.00(16-33)$ & $<0.001$ \\
\hline $\mathrm{HDL}-\mathrm{C}(\mathrm{mmol} / \mathrm{L})$ & $1.73(0.39)$ & $1.62(0.38)$ & $1.48(0.36)$ & $1.32(0.31)$ & $1.15(0.27)$ & $<0.001$ \\
\hline TC (mmol/L) & $4.73(0.78)$ & $4.99(0.81)$ & $5.11(0.86)$ & $5.28(0.82)$ & $5.50(0.88)$ & $<0.001$ \\
\hline TG $(\mathrm{mmol} / \mathrm{L})$ & $0.41(0.30-0.53)$ & $0.56(0.44-0.73)$ & $0.71(0.55-0.93)$ & $0.95(0.72-1.24)$ & $1.42(1.03-1.94)$ & $<0.001$ \\
\hline $\mathrm{FPG}(\mathrm{mmol} / \mathrm{L})$ & $4.88(0.37)$ & $5.03(0.38)$ & $5.16(0.38)$ & $5.27(0.36)$ & $5.40(0.36)$ & $<0.001$ \\
\hline TyG & $7.33(0.45)$ & $7.73(0.39)$ & $7.98(0.40)$ & $8.29(0.41)$ & $8.72(0.49)$ & $<0.001$ \\
\hline HbA1c (\%) & $5.10(4.90-5.35)$ & $5.10(4.91-5.40)^{* * *}$ & $5.10(4.90-5.40)$ & $5.20(5.00-5.40)$ & $5.20(5.00-5.50)$ & $<0.001$ \\
\hline SBP $(\mathrm{mmHg})$ & $104.98(12.14)$ & 108.99 (12.62) & $113.40(12.96)$ & $117.90(13.62)$ & $124.40(14.44)$ & $<0.001$ \\
\hline $\mathrm{DBP}(\mathrm{mmHg})$ & $64.91(8.30)$ & $67.66(9.06)$ & $70.55(9.22)$ & $74.01(9.56)$ & $78.48(9.93)$ & $<0.001$ \\
\hline Drinking status & & & & & & $<0.001$ \\
\hline Non or small & 2606 (91.44\%) & 2451 (86.00\%) & 2305 (80.85\%) & 2197 (77.11\%) & 2246 (78.78\%) & \\
\hline Light & $209(7.33 \%)$ & 307 (10.77\%) & 406 (14.24\%) & $434(15.23 \%)$ & $402(14.10 \%)$ & \\
\hline Moderate & $35(1.23 \%)$ & 92 (3.23\%) & $140(4.91 \%)$ & 218 (7.65\%) & 203 (7.12\%) & \\
\hline Smoking status & & & & & & $<0.001$ \\
\hline Non & 2299 (80.67\%) & 2018 (70.81\%) & 1732 (60.75\%) & 1387 (48.68\%) & 1310 (45.95\%) & \\
\hline Former & $246(8.63 \%)$ & 406 (14.25\%) & $529(18.55 \%)$ & 690 (24.22\%) & 688 (24.13\%) & \\
\hline Current & $305(10.70 \%)$ & $426(14.95 \%)$ & 590 (20.69\%) & 772 (27.10\%) & 853 (29.92\%) & \\
\hline
\end{tabular}

Values were expressed as mean (SD) or medians (quartile interval) or $\mathrm{n}(\%)$. Abbreviations: NAFLD: Nonalcoholic fatty liver disease; BMI body mass index; TyG the triglyceride-glucose index; TyG-BMI triglyceride glucose-body mass index; WC Waist circumference; ALT alanine aminotransferase; $A S T$ aspartate aminotransferase; GGT gamma-glutamyl transferase; $H D L-C$ high-density lipoprotein cholesterol; TC total cholesterol; TG triglyceride; HbA1c hemoglobin A1c; FPG fasting plasma glucose; SBP systolic blood pressure; $D B P$ Diastolic blood pressure. ${ }^{*} P>0.05$ vs Q5. ${ }^{* *} P>0.05$ vs $Q 2$. ${ }^{* *} P>0.05$ vs $Q 1, Q 3$. Other variables with no special mark on the upper right corner had $P$ values $<0.05$ after pair-wise comparison

related to relationship in the general population; and with the gradual increase of TyG-BMI, the NAFLD risk was gradually increased (Table 2). In multivariate analysis, after adjusting the non-collinear general demographic data in model 1 , the positive correlation and trend between the TyG-BMI and NAFLD remained unchanged (OR: 5.40 per SD increase; 95\% CI: 5.01 to 5.83; $P$-trend $<0.00001)$. Model 2 adjusted the non-collinear variables in which the impact of the TyG-BMI on NAFL $\mathrm{D}$ risk was more than $10 \%$ and the non-collinear variables in univariate analysis with $P$ value less than 0.05 . The OR values of the TyG-BMI and NAFLD were slightly lower than those in model 1 , and the positive correlation direction and trend of the core results were maintained (OR: 3.87 per SD increase; 95\% CI: 3.51 to 4.26; $P$-trend $<0.00001)$. In model 3 , after adjusting the non-collinear variables related to the TyG-BMI, it was found that the NAFLD risk increased by $290 \%$ for per 1 SD increase in TyG-BMI (95\% CI: 3.54 to 4.29; $P$-trend< 0.00001). In four different models, the core direction of the relationship between NAFLD and the TyG-BMI did not change significantly, and the results of sensitivity analysis further verified the stability of the positive correlation between them. 
Table 2 Logistic regression analyses for the association between TyG-BMI and incident NAFLD in different models

\begin{tabular}{|c|c|c|c|c|}
\hline & \multicolumn{4}{|c|}{ Odds ratios ( $95 \%$ confidence interval) } \\
\hline & Crude model & Model 1 & Model 2 & Model 3 \\
\hline TyG-BMI(per SD increase) & $6.17(5.75,6.62)$ & $5.40(5.01,5.83)$ & $3.87(3.51,4.26)$ & $3.90(3.54,4.29)$ \\
\hline \multicolumn{5}{|l|}{ TyG-BMI (Quintile) } \\
\hline Quintile 1 & Ref & Ref & Ref & Ref \\
\hline Quintile 2 & $5.17(2.70,9.89)$ & $4.25(2.22,8.15)$ & $4.04(2.03,8.03)$ & $4.03(2.03,8.01)$ \\
\hline Quintile 3 & $19.26(10.47,35.43)$ & $13.33(7.22,24.60)$ & $10.72(5.58,20.62)$ & $10.81(5.63,20.75)$ \\
\hline Quintile 4 & $70.61(38.80,128.51)$ & $42.25(23.09,77.30)$ & $25.09(13.11,47.99)$ & $25.52(13.36,48.74)$ \\
\hline Quintile 5 & $344.54(189.71,625.75)$ & $186.82(102.20,341.51)$ & $72.84(37.82,140.30)$ & $74.76(38.86,143.79)$ \\
\hline$P$-trend & $<0.0001$ & $<0.0001$ & $<0.0001$ & $<0.0001$ \\
\hline
\end{tabular}

Abbreviations: TyG-BMI: triglyceride glucose-body mass index;

Model 1 adjusted for sex, age, habits of exercise, drinking status. Smoking status, SBP, and height;

Model 2 adjusted for sex, age, ALT, AST, habits of exercise, GGT; HDL-C, TC, TG, FPG, HbA1c, smoking status, SBP and height;

Model 3 adjusted for sex, age, ALT, AST, habits of exercise, GGT; HDL-C, TC, TG, FPG, HbA1c, smoking status, drinking status, SBP and height

\section{Non-linear relationship analysis between NAFLD and the TyG-BMI}

To further explore TyG-BMI and non-linear relation between NAFLD, the team performed smoothing function analysis using a generalized additive model to simulate the potential non-linear association between NAFLD and the TyG-BMI (Fig. 1). Before and after adjustment, the model maintained the stability of the non-linear association between the TyG-BMI and NAFLD, and threshold and saturation effects were found in the correlation between NAFLD and the TyG-BMI. A TyGBMI between 100 and 150 likely indicates a threshold effect point, and a TyG-BMI between 300 and 400 suggests the corresponding NAFLD risk may indicate a saturation effect.

\section{Subgroup analysis}

In subgroup analysis (Table 3), a significant interaction was found between age, the BMI and the TyG-BMIrelated NAFLD risk $(P$-interaction $<0.05)$, while the interaction between sex, the smoking status, habit of exercise, the drinking status and the TyG-BMI indicated no significant significance. Age stratification analysis showed that middle-aged people had a higher risk of TyG-BMI-related NAFLD than other age groups; BMI stratification also found an interesting result: among non-obese people, the risk of NAFLD related to the TyG-BMI was higher than that of overweight and obese people.

\section{ROC analysis}

Figure 2 shows the ROC curve of the ability of the TyG$\mathrm{BMI}$ and traditional indicators to predict NAFLD risk. Table 4 shows the results of the area under the curve (AUC) in ROC analysis, in which the AUC of the TyGBMI was 0.886 (95\% CI: 0.8797-0.8927), significantly higher than that of the single TyG index, TG/HDL ratio, the BMI, and traditional markers such as TG, FPG, WC, HDL-C, HbA1c, and TC $(P<0.0001)$. Furthermore, TyG-WC showed that the area under the ROC curve to
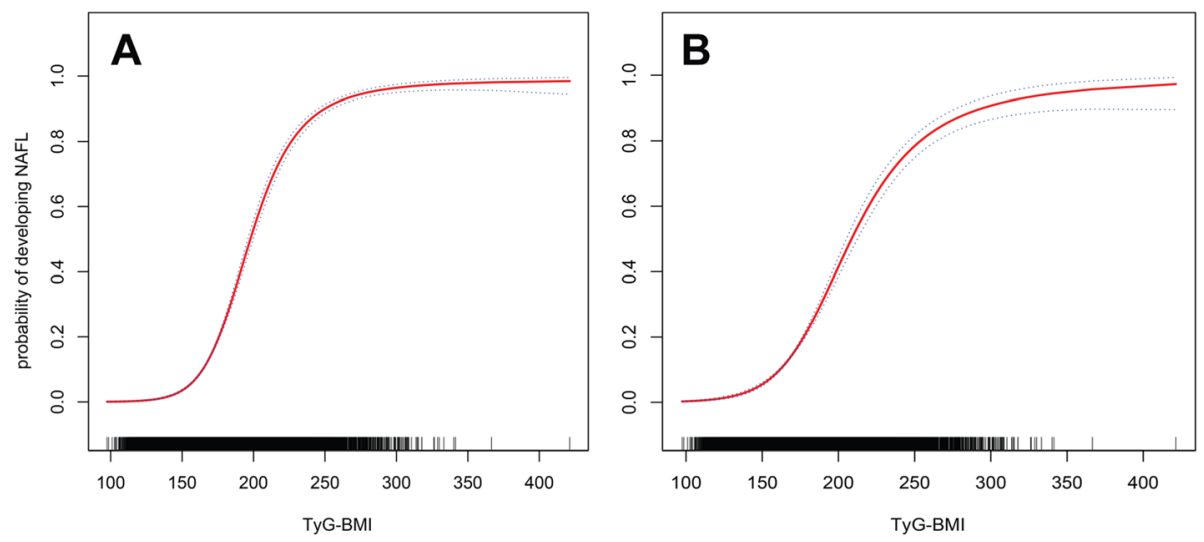

Fig. 1 Non-linear relationship between the TyG-BMI and NAFLD in the unadjusted model (A) and adjusted model (B). Dashed lines indicate 95\% confidence intervals. Adjusted for sex, age, ALT, AST, habits of exercise, GGT, HDL-C, TC, TG, FPG, HbA1c, smoking status, SBP and height 
Table 3 Stratified associations between TyG-BMI and NAFLD by age, sex, habit of exercise, BMI, smoking status and drinking status

\begin{tabular}{|c|c|c|c|c|}
\hline Subgroup & No. of participants & unadjusted OR (95\% Cl) & adjusted OR (95\% Cl) & $P$-interaction \\
\hline Age (years) & & & & 0.0095 \\
\hline $18-30$ & 645 & $6.12(4.04,9.27)$ & $3.68(2.35,5.76)$ & \\
\hline $31-45$ & 8210 & $6.98(6.33,7.70)$ & $4.31(3.82,4.87)$ & \\
\hline $46-60$ & 4909 & $5.14(4.60,5.74)$ & $3.30(2.90,3.76)$ & \\
\hline$>60$ & 487 & $5.35(3.59,7.98)$ & $3.86(2.53,5.89)$ & \\
\hline Sex & & & & 0.1101 \\
\hline Male & 7411 & $5.50(5.04,6.00)$ & $3.68(3.28,4.12)$ & \\
\hline Female & 6840 & $5.96(5.25,6.76)$ & $4.20(3.64,4.83)$ & \\
\hline Habit of exercise & & & & 0.0667 \\
\hline Yes & 2470 & $6.88(5.71,8.30)$ & $4.58(3.72,5.64)$ & \\
\hline No & 11,781 & $6.04(5.60,6.52)$ & $3.76(3.41,4.16)$ & \\
\hline BMI $\left(\mathrm{kg} / \mathrm{m}^{2}\right)$ & & & & 0.0002 \\
\hline$<24$ & 10,881 & $10.02(8.65,11.59)$ & $5.82(4.75,7.14)$ & \\
\hline$\geq 24,<28$ & 2726 & $4.58(3.86,5.44)$ & $2.81(2.21,3.56)$ & \\
\hline$\geq 28$ & 644 & $3.97(2.79,5.66)$ & $2.89(1.97,4.25)$ & \\
\hline Smoking status & & & & 0.5652 \\
\hline Non & 8746 & $6.59(5.98,7.27)$ & $4.01(3.56,4.51)$ & \\
\hline Former & 2559 & $5.78(4.95,6.75)$ & $3.74(3.13,4.47)$ & \\
\hline Current & 2946 & $5.46(4.75,6.29)$ & $3.66(3.12,4.30)$ & \\
\hline Drinking status & & & & 0.5258 \\
\hline Non or small & 11,805 & $6.43(5.95,6.96)$ & $3.95(3.57,4.37)$ & \\
\hline Light & 1758 & $5.45(4.47,6.64)$ & $3.47(2.78,4.33)$ & \\
\hline Moderate & 688 & $5.88(4.21,8.21)$ & $4.05(2.83,5.80)$ & \\
\hline
\end{tabular}

Abbreviations: OR Odds ratios; other abbreviations as in Table 1

Adjusted for sex, age, ALT, AST, habits of exercise, GGT; HDL-C, TC, TG, FPG, HbA1c, smoking status, SBP and height

detect NAFLD was second only to the TyG-BMI, and no significant difference was found between them $(P=$ 0.3662). Considering that significant differences were found in age and BMI stratification in subgroup analysis, the predictive performance of the TyG-BMI was further evaluated in different age stratifications and BMI stratifications for NAFLD by ROC analysis, revealing that the TyG-BMI had a larger AUC to predict NAFLD in young and middle-aged and non-obese people (Table 5). Additionally, studies on TyG-WC also used the same analysis steps, demonstrating that TyG-WC also had a high predictive value for NAFLD in non-obese people and young and middle-aged people.

\section{Discussion}

NAFLD not only causes a series of pathological changes of the liver, but also leads to the occurrence and development of a variety of extrahepatic diseases, which has become an important risk factor for a variety of metabolism-related diseases [2, 5-7]. This study reported for the first time a stable and independent positive association between NAFLD and the TyG-BMI in the general population that is not simply linear but nonlinear and has threshold and saturation effects.

\section{Comparison with other studies}

IR is the key factor in the pathogenesis of NAFLD $[8,9]$, in which blood lipid parameters, the blood lipid ratio, blood glucose parameters, the BMI and WC were often used to evaluate the IR status. Recently, an increasing number of studies have found that the combination of FPG and TG (TyG) can better identify IR, likely related to the TyG index considering both blood glucose and blood lipids, and the TyG index reflecting the metabolic status of the body more comprehensively [10-12]. The $\mathrm{BMI}$ is the most important anthropometric index commonly used to evaluate obesity clinically. In 2016, Er et al. found that the new index formed by combining the TyG index with the BMI (TyG-BMI) can better reflect the state of IR. Compared with lipid parameters, the lipid ratio, blood glucose parameters, the TyG index and obesity-related parameters, the TyG-BMI has the largest AUC in identifying IR [13]. In several follow-up studies, the Tuo and Lim teams have confirmed this 


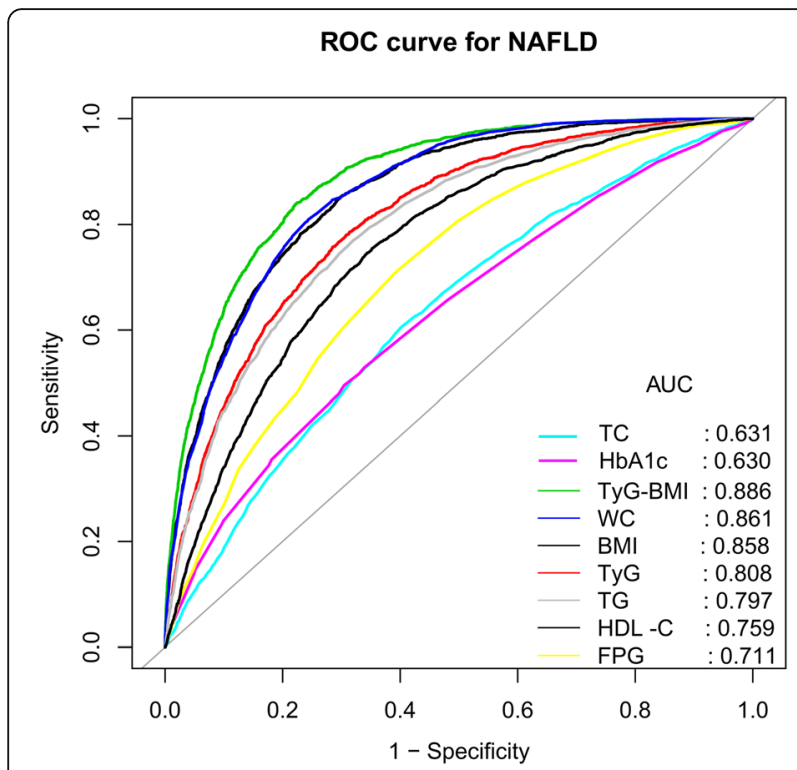

Fig. 2 ROC curve analysis of NAFLD-related indicators. Receiver operating characteristic (ROC), NAFLD: nonalcoholic fatty liver disease; BMI: body mass index; TyG: triglyceride-glucose index; TyGBMI: triglyceride glucose-body mass index; WC: waist circumference; HDL-C: high-density lipoprotein cholesterol; TC: total cholesterol; TG: triglyceride; HbA1c: haemoglobin A1c; FPG: fasting plasma glucose

finding $[27,28]$. Additionally, recently, the TyG-BMI has been found to be strongly associated with cardiovascular and cerebrovascular diseases such as hypertension and ischaemic stroke $[29,30]$. On the other hand, the TyG-BMI also has a similar positive correlation with NAFLD and shows a better predictive performance than other traditional indicators [14-16]. In a cross-sectional survey of 6809 healthy non-obese people, Zhang et al. first found that TyG-BMI is positively correlated with NAFLD and can better identify non-obese NAFLD than the TyG index, BMI, TG and FPG [14]. This finding suggests that the TyG-BMI may be a useful marker to predict NAFLD and may provide benefits to prevent and treat NAFLD. Followup studies by Li et al. also confirmed this finding that the TyG-BMI can be used to identify the NAFLD risk in non-obese people and may be at a higher risk in women [15]. In addition, a recent study by Khamseh et al. found that the TyG-WC and TyG-BMI are associated with NAFLD in overweight and obese people, and the correlation between liver fibrosis and TyGBMI was stronger [16]. Compared with these similar studies, this study considered non-obese individuals, overweight individuals and obese individuals in the general population and continued to expand the sample size based on previous studies. The results of this study not only verified the correlation between NAFL $\mathrm{D}$ and the TyG-BMI but also found in further ROC analysis that TyG-BMI had the best NAFLD discrimination ability compared with the HbA1c, TG, HDLC, TyG index, FPG, BMI, WC, TG/HDL-C ratio, TyG-WC and TC. Importantly, the TyG-BMI shows excellent predictive performance in detecting NAFLD in young and middle-aged people (18-30 years: AUC: $0.9258 ; 95 \%$ CI: $0.8908-0.9608 ; 31-45$ years: AUC: 0.9090; 95\% CI: 0.9015-0.9164). More importantly, the TyG-BMI is calculated according to the FPG, TG and BMI [13], which can be obtained clinically with simple calculation, is inexpensive and convenient, and conducive to rapid promotion and application in clinical practice.

Compared with previous studies, this study also explored the non-linear correlation, and the results were equally surprising. The results show a stable non-linear relationship between NAFLD and the TyG-BMI before and after adjustment of the generalized additive model.

Table 4 Areas under the receiver operating characteristic curves for each evaluated parameters in identifying nonalcoholic fatty liver disease

\begin{tabular}{|c|c|c|c|c|c|}
\hline & AUC & $95 \%$ confidence interval & Best threshold & Specificity & Sensitivity \\
\hline BMl & $0.858^{*}$ & $0.8503-0.8651$ & 22.5521 & 0.7015 & 0.8524 \\
\hline TyG & $0.808^{*}$ & $0.7996-0.8173$ & 8.2059 & 0.7149 & 0.7575 \\
\hline TyG-BMI & 0.886 & $0.8797-0.8927$ & 189.6932 & 0.7787 & 0.8381 \\
\hline WC & $0.861^{*}$ & $0.8539-0.8681$ & 79.6500 & 0.7571 & 0.8085 \\
\hline TyG-WC & 0.885 & $0.8782-0.8911$ & 637.8369 & 0.7207 & 0.8827 \\
\hline TC & $0.631^{*}$ & $0.6191-0.6427$ & 5.2108 & 0.5985 & 0.6051 \\
\hline $\mathrm{HbA1c}$ & $0.630^{*}$ & $0.6175-0.6419$ & 5.3250 & 0.6956 & 0.4950 \\
\hline FPG & $0.711^{*}$ & $0.7005-0.7217$ & 5.1902 & 0.6073 & 0.7100 \\
\hline TG & $0.797^{*}$ & $0.7877-0.8061$ & 0.8411 & 0.6799 & 0.7698 \\
\hline $\mathrm{HDL}-\mathrm{C}$ & $0.759^{*}$ & $0.7490-0.7686$ & 1.3408 & 0.6478 & 0.7547 \\
\hline TG/HDL-C & $0.815^{*}$ & $0.8060-0.8233$ & 0.6119 & 0.6818 & 0.8046 \\
\hline
\end{tabular}

Abbreviations: AUC area under the curve; other abbreviations as in Table 1. ${ }^{*} P<0.0001$, compare with TyG-BMI 
Table 5 Results of the receiver operating characteristic curve analyses of TyG-BMI and TyG-WC for predicting NAFLD in subgroups of age and BMI

\begin{tabular}{|c|c|c|c|c|c|c|}
\hline & AUC & $95 \% \mathrm{Cl}$ low & $95 \% \mathrm{Cl}$ upp & Best threshold & Specificity & Sensitivity \\
\hline \multicolumn{7}{|l|}{ TyG-BMI } \\
\hline \multicolumn{7}{|l|}{ Age (years) } \\
\hline$>60$ & 0.8247 & 0.7778 & 0.8715 & 196.7369 & 0.7956 & 0.7531 \\
\hline $46-60$ & 0.8440 & 0.8311 & 0.8569 & 196.4398 & 0.7978 & 0.7271 \\
\hline $31-45$ & 0.9090 & 0.9015 & 0.9164 & 189.6580 & 0.8057 & 0.8714 \\
\hline $18-30$ & 0.9258 & 0.8908 & 0.9608 & 172.4438 & 0.7599 & 0.9268 \\
\hline \multicolumn{7}{|l|}{ BMI $\left(\mathrm{kg} / \mathrm{m}^{2}\right)$} \\
\hline$\geq 28$ & 0.7150 & 0.6696 & 0.7604 & 254.8981 & 0.7296 & 0.6000 \\
\hline$\geq 24,<28$ & 0.7070 & 0.6876 & 0.7264 & 216.5987 & 0.7008 & 0.5998 \\
\hline$<24$ & 0.8457 & 0.8335 & 0.8579 & 178.0661 & 0.7658 & 0.7776 \\
\hline \multicolumn{7}{|l|}{ TyG-WC } \\
\hline \multicolumn{7}{|l|}{ Age (years) } \\
\hline$>60$ & 0.7942 & 0.7439 & 0.8445 & 685.9048 & 0.7537 & 0.7037 \\
\hline $46-60$ & 0.8426 & 0.8298 & 0.8554 & 666.8453 & 0.7436 & 0.7818 \\
\hline $31-45$ & 0.9080 & 0.9006 & 0.9154 & 625.1197 & 0.7995 & 0.8621 \\
\hline $18-30$ & 0.9393 & 0.9110 & 0.9676 & 619.1378 & 0.8493 & 0.9024 \\
\hline \multicolumn{7}{|l|}{$\mathrm{BMI}\left(\mathrm{kg} / \mathrm{m}^{2}\right)$} \\
\hline$\geq 28$ & 0.7346 & 0.6891 & 0.7800 & 806.3716 & 0.7547 & 0.6186 \\
\hline$\geq 24,<28$ & 0.7099 & 0.6906 & 0.7292 & 711.0072 & 0.6313 & 0.6988 \\
\hline$<24$ & 0.8528 & 0.8413 & 0.8643 & 620.9366 & 0.7500 & 0.8153 \\
\hline
\end{tabular}

Abbreviations: AUC area under the curve; other abbreviations as in Table 1

It is exciting that threshold and saturation effects exist in the correlation between NAFLD and the TyG-BMI; a TyG-BMI between 100 and 150 likely indicates a threshold effect point, while a TyG-BMI between 300 and 400 likely suggests that the corresponding NAFLD risk is a saturation effect. As far as I know, this is the first report of the non-linear relationship between NAFLD and the TyG-BMI. The discovery of the threshold effect and saturation effect can provide new ideas for clinical medical workers to prevent and treat NAFLD.

In subgroup analysis, some interesting phenomena were also found in this study. Compared with other age groups, middle-aged people have a higher risk of TyG-BMI-related NAFLD. After further analysis of the baseline information of the study population grouped according to age, middle-aged people were found to lack physical exercise compared with other age groups [14.37\% (31-45 years) vs $18.29 \%$ ( $18-30$ years), $20.55 \%$ (46-60 years), and $>33.47 \%$ (60 years); Supplementary Table 3]. Presently, exercise is the only recognized method to prevent and treat NAFLD [8, 31, 32]. A lack of physical exercise can not only easily lead to NAFLD but also easily lead to disorders of blood lipids, blood pressure and blood glucose metabolism and then increase the risk of cardiovascular and cerebrovascular diseases, diabetes and neuropsychiatric diseases [33-35]. The cause of this situation may be related to the current social structure and division of labour. With the further aggravation of ageing and growing economic needs of families, the pressure on middle-aged people is also increasing [36, 37]. In addition to age, a significant interaction was found between the BMI and TyG-BMI, in which the risk of NAFLD associated with TyG-BMI was higher in non-obese people than in overweight and obese people. After further analysis of the baseline information of subjects for BMI stratification (Supplementary Table 4), it can be seen that there were more non-obese women than men in this study. Sex differences in non-obese NAFLD have also been noted in some previous studies [38, 39], likely related to the definition of non-obesity being mainly distinguished by the BMI. Generally, female individuals tend to have more subcutaneous and visceral fat $[40,41]$, and the BMI alone does not provide a complete picture of this information [39]. Recently, related studies on non-obese NAFLD have found that non-obese people are more prone to metabolic disorders $[42,43]$. Combined with the subgroup analysis content of this study, the research team agreed that non-obese NAFLD should be given more attention. 


\section{Study strengths and limitations}

Several strengths worth mentioning in this research. 1) This report is the first to study TyG-BMI and the risk of NAFLD in the general population. The population of this study is not only limited to non-obese people but also overweight and obese people. The research data of the general population provide more reference information for follow-up research. 2) This report is also the first to show a non-linear relationship between NAFLD and the TyG-BMI with threshold and saturation effects between them, a finding that will be very helpful help to clinicians. 3) This study included 14,251 subjects, representing a large sample size, and performed using strict statistical adjustment and sensitivity analysis. The results of the data analysis are considered relatively stable.

In addition to research strengths, some research limitations should be highlighted. 1) The design of this study was cross-sectional. Thus, it can only explain the correlation between NAFLD and the TyG-BMI; the causal association between the two must be confirmed by further longitudinal studies. Inevitably, some covariables cannot be measured or evaluated in observational studies; thus, residual confounding may occur [44]. However, this study fully adjusted the variables under the existing conditions, and the main results were similar to previous research results [14-16]. Therefore, the results of this study can be considered to be relatively stable. 2) The diagnosis of NAFLD in this study was conducted according to abdominal ultrasound, while the gold standard for the diagnosis of NAFLD was liver biopsy [1, 2], leading to some patients not having mild hepatic steatosis. However, for more than thousands or even tens of thousands of subjects, liver biopsies would be unethical. 3) Measurement data of IR were lacking in this study, and IR may be the key factor leading to NAFLD with a higher TyG-BMI. Although TyG-BMI has better predictive performance than the TyG index in identifying IR by reviewing the historical literature [13, 27, 28], this conclusion has not been verified in this study; 4) the results of this study are primarily applicable to the assessment of the risk of NAFLD in the general population, but can not further distinguish between nonalcoholic steatohepatitis and NAFL.

\section{Conclusion}

Overall, this study confirmed a statistically significant correlation between the increase in the TyG-BMI and NAFLD risk in the general population. A TyG-BMI between 100 and 150 likely indicates a threshold effect point, while a TyG-BMI between 300 and 400 likely suggests that the corresponding NAFLD risk is saturated. The present study further enhanced the application value of the TyG-BMI in the diagnosis of NAFLD and indicated a simpler and more economical method to prevent and treat NAFLD.

\section{Abbreviations}

WC: Waist circumference; AUC: Area under the curve; IR: Insulin resistance; GGT: Gamma-glutamyl transferase; Cl: Confidence interval; FPG: Fasting blood glucose; S/BBP: Systolic/diastolic blood pressure; BMI: Body mass index; ALT: Alanine aminotransferase; HbA1c: Hemoglobin A1c; AST: Aspartate aminotransferase; TyG index: Triglyceride-glucose index; TC: Total cholesterol: SD: Standard deviation; TG: Triglyceride; OR: Odds ratio; TyG-BMI: Triglyceride glucose-body mass index; NAFLD: Nonalcoholic fatty liver disease; HDL-

C: High-density lipoprotein cholesterol

\section{Supplementary Information}

The online version contains supplementary material available at https://doi. org/10.1186/s12944-021-01506-9.

Additional file 1: Supplementary Table 1. Collinearity diagnostics steps. Supplementary Table 2. Association between TyG-BMI and baseline variables. Supplementary Table 3. Baseline characteristics of age groups. Supplementary Table 4. Baseline characteristics of BMl groups.

\section{Acknowledgements}

We thank all participants in this study.

\section{Authors' contributions}

Conceptualization and research design: Guobo Xie and Rongsheng Wang; Project administration: Guobo Xie, Longlong DAl and Rongsheng Wang; Supervision: Guobo Xie and Yanjia Zhong; Writing-Original draft preparation: Guobo Xie, Longlong DAl, Yanjia Zhong and Rongsheng Wang; WritingReviewing and Editing: Guobo Xie, Longlong DAl, Yanjia Zhong and Rongsheng Wang. The author(s) read and approved the final manuscript.

\section{Funding}

Not applicable.

\section{Availability of data and materials}

The datasets that support the conclusions of this article can be found in the Dryad repository.

\section{Declarations}

Ethics approval and consent to participate

Because Murakami Memorial Hospital's institutional ethics committee had authorized the previous study, separate ethical approval was no longer required for this study. Additionally, because the identification information of the subjects was deleted from the data and uniformly replaced by the health check code and the informed consent for each subject on using the data in the previous study was obtained, the application to obtain informed consent again was not required in this study.

Consent for publication

Not applicable.

\section{Competing interests}

The author declares that the study was conducted without any commercial or financial relationship.

\section{Author details}

'Department of Intensive Care Unit, Jiangxi Provincial People's Hospital Affiliated to Nanchang University, Nanchang, P.R. China 330006. '2Department of Cardiology, Ruichang People's Hospital, Jiujiang, P.R. China 332200. ${ }^{3}$ Department of Endocrinology, Jiangxi Provincial People's Hospital Affiliated to Nanchang University, Nanchang, P.R. China 330006. ${ }^{4}$ Department of Cardiology, Jiangxi Provincial People's Hospital Affiliated to Nanchang University, Nanchang, P.R. China 330006. 
Received: 23 June 2021 Accepted: 15 July 2021

Published online: 28 July 2021

\section{References}

1. Neuschwander-Tetri BA. Non-alcoholic fatty liver disease. BMC Med. 2017; 15(1):45. https://doi.org/10.1186/s12916-017-0806-8.

2. Younossi ZM, Koenig AB, Abdelatif D, Fazel Y, Henry L, Wymer M. Global epidemiology of nonalcoholic fatty liver disease-meta-analytic assessment of prevalence, incidence, and outcomes. Hepatology. 2016 Jul;64(1):73-84. https://doi.org/10.1002/hep.28431.

3. Estes C, Razavi H, Loomba R, Younossi Z, Sanyal AJ. Modeling the epidemic of nonalcoholic fatty liver disease demonstrates an exponential increase in burden of disease. Hepatology. 2018;67(1):123-33. https://doi.org/10.1002/ hep.29466.

4. Bedossa P. Pathology of non-alcoholic fatty liver disease. Liver Int. 2017;37: 85-9. https://doi.org/10.1111/liv.13301.

5. Byrne CD, Targher G. NAFLD: a multisystem disease. J Hepatol. 2015;62(1): S47-64. https://doi.org/10.1016/j.jhep.2014.12.012.

6. Adams LA, Anstee QM, Tilg H, Targher G. Non-alcoholic fatty liver disease and its relationship with cardiovascular disease and other extrahepatic diseases. Gut. 2017;66(6):1138-53. https://doi.org/10.1136/ gutjnl-2017-313884.

7. Ballestri S, Mantovani A, Nascimbeni F, Lugari S, Lonardo A. Extra-hepatic manifestations and complications of nonalcoholic fatty liver disease. Future Med Chem. 2019;11(16):2171-92. https://doi.org/10.4155/fmc-2019-0003.

8. Wang XJ, Malhi H. Nonalcoholic fatty liver disease. Ann Intern Med. 2018; 169(9):ITC65-80. https://doi.org/10.7326/AITC201811060.

9. Khan RS, Bril F, Cusi K, Newsome PN. Modulation of insulin resistance in nonalcoholic fatty liver disease. Hepatology. 2019;70(2):711-24. https://doi. org/10.1002/hep.30429.

10. Guerrero-Romero F, Simental-Mendía LE, González-Ortiz M, MartínezAbundis E, Ramos-Zavala MG, Hernández-González SO, et al. The product of triglycerides and glucose, a simple measure of insulin sensitivity. Comparison with the euglycemic-hyperinsulinemic clamp. J Clin Endocrinol Metab. 2010;95(7):3347-51. https://doi.org/10.1210/jc.2010-0288.

11. Sánchez-García A, Rodríguez-Gutiérrez R, Mancillas-Adame L, González-Nava V, Díaz González-Colmenero A, Solis RC, et al. Diagnostic accuracy of the triglyceride and glucose index for insulin resistance: a systematic review. Int J Endocrinol. 2020;2020:4678526.

12. Dikaiakou E, Vlachopapadopoulou EA, Paschou SA, Athanasouli F, Panagiotopoulos I, Kafetzi M, et al. Triglycerides-glucose (TyG) index is a sensitive marker of insulin resistance in Greek children and adolescents. Endocrine. 2020;70(1):58-64. https://doi.org/10.1007/s12020-020-02374-6.

13. Er LK, Wu S, Chou HH, Hsu LA, Teng MS, Sun YC, et al. Triglyceride glucosebody mass index is a simple and clinically useful surrogate marker for insulin resistance in nondiabetic individuals. PLoS One. 2016;11(3):e0149731. https://doi.org/10.1371/journal.pone.0149731.

14. Zhang S, Du T, Li M, Jia J, Lu H, Lin X, et al. Triglyceride glucose-body mass index is effective in identifying nonalcoholic fatty liver disease in nonobese subjects. Medicine (Baltimore). 2017;96:e7041.

15. Li Y, Zheng R, Li J, Feng S, Wang L, Huang Z. Association between triglyceride glucose-body mass index and non-alcoholic fatty liver disease in the non-obese Chinese population with normal blood lipid levels: a secondary analysis based on a prospective cohort study. Lipids Health Dis. 2020;19(1):229. https://doi.org/10.1186/s12944-020-01409-1.

16. Khamseh ME, Malek M, Abbasi R, Taheri H, Lahouti M, Alaei-Shahmiri F. Triglyceride glucose index and related parameters (triglyceride glucosebody mass index and triglyceride glucose-waist circumference) identify nonalcoholic fatty liver and liver fibrosis in individuals with overweight/ obesity. Metab Syndr Relat Disord. 2021;19(3):167-73. https://doi.org/10.1 089/met.2020.0109.

17. Okamura T, Hashimoto Y, Hamaguchi M, Obora A, Kojima T, et al. Ectopic fat obesity presents the greatest risk for incident type 2 diabetes: a population-based longitudinal study. Int J Obes (Lond). 2019;43:139-48.

18. Okamura T, et al. Data from: ectopic fat obesity presents the greatest risk for incident type 2 diabetes: a population-based longitudinal study, Dryad, Dataset; 2019. https://doi.org/10.5061/dryad.8q0p192.

19. Choi JH, Sohn W, Cho YK. The effect of moderate alcohol drinking in nonalcoholic fatty liver disease. Clin Mol Hepatol. 2020;26(4):662-9. https:// doi.org/10.3350/cmh.2020.0163.
20. Hamaguchi M, Kojima T, Itoh Y, Harano Y, Fujii K, Nakajima T, et al. The severity of ultrasonographic findings in nonalcoholic fatty liver disease reflects the metabolic syndrome and visceral fat accumulation. Am J Gastroenterol Am J Gastroenterol. 2007;102(12):2708-15. https://doi.org/1 $0.1111 /$ j.1572-0241.2007.01526.x.

21. Kim JH. Multicollinearity and misleading statistical results. Korean J Anesthesiol. 2019;72(6):558-69. https://doi.org/10.4097/kja.19087.

22. Sheng G, Peng N, Hu C, Zhong L, Zhong M, Zou Y. The albumin-to-alkaline phosphatase ratio as an independent predictor of future non-alcoholic fatty liver disease in a 5-year longitudinal cohort study of a non-obese Chinese population. Lipids Health Dis. 2021;20(1):50. https://doi.org/10.1186/s12944021-01479-9.

23. Fitchett EJA, Seale AC, Vergnano S, Sharland M, Heath PT, Saha SK, et al. Strengthening the reporting of observational studies in epidemiology for newborn infection (STROBE-NI): an extension of the STROBE statement for neonatal infection research. Lancet Infect Dis. 2016;16(10):e202-13. https:// doi.org/10.1016/S1473-3099(16)30082-2.

24. Vandenbroucke JP, von Elm E, Altman DG, Gøtzsche PC, Mulrow CD, Pocock SJ, et al. Strengthening the reporting of observational studies in epidemiology (STROBE): explanation and elaboration. Int J Surg. 2014;12(12): 1500-24. https://doi.org/10.1016/j.ijsu.2014.07.014.

25. Zou Y, Zhong L, Hu C, Zhong M, Peng N, Sheng G. LDL/HDL cholesterol ratio is associated with new-onset NAFLD in Chinese non-obese people with normal lipids: a 5-year longitudinal cohort study. Lipids Health Dis. 2021;20(1):28. https://doi.org/10.1186/s12944-021-01457-1.

26. Fan N, Peng L, Xia Z, Zhang L, Song Z, Wang Y, et al. Triglycerides to highdensity lipoprotein cholesterol ratio as a surrogate for nonalcoholic fatty liver disease: a cross-sectional study. Lipids Health Dis. 2019;18(1):39. https:// doi.org/10.1186/s12944-019-0986-7.

27. Tuo X, Yuan J, Wang XH, Xin Z. Identifying the insulin resistance index in nondiabetic Chinese subjects. Medicine (Baltimore). 2020;99:e19023.

28. Lim J, Kim J, Koo SH, Kwon GC. Comparison of triglyceride glucose index, and related parameters to predict insulin resistance in Korean adults: an analysis of the 2007-2010 Korean National Health and nutrition examination survey. PLoS One. 2019;14(3):e0212963. https://doi.org/10.1371/journal.pone. 0212963.

29. Zeng ZY, Liu SX, Xu H, Xu X, Liu XZ, Zhao XX. Association of triglyceride glucose index and its combination of obesity indices with prehypertension in ean individuals: a cross-sectional study of Chinese adults. J Clin Hypertens (Greenwich). 2020;22(6):1025-32. https://doi.org/10.1111/jch.13878.

30. Du Z, Xing L, Lin M, Sun Y. Estimate of prevalent ischemic stroke from triglyceride glucose-body mass index in the general population. BMC Cardiovasc Disord. 2020;20(1):483. https://doi.org/10.1186/s12872-020-01768-8.

31. Keating SE, George J, Johnson NA. The benefits of exercise for patients with non-alcoholic fatty liver disease. Expert Rev Gastroenterol Hepatol. 2015; 9(10):1247-50. https://doi.org/10.1586/17474124.2015.1075392.

32. Golabi P, Locklear CT, Austin P, Afdhal S, Byrns M, Gerber L, et al. Effectiveness of exercise in hepatic fat mobilization in non-alcoholic fatty liver disease: systematic review. World J Gastroenterol. 2016;22(27):6318-27. https://doi.org/10.3748/wjg.v22.i27.6318.

33. Pedersen BK, Saltin B. Exercise as medicine - evidence for prescribing exercise as therapy in 26 different chronic diseases. Scand J Med Sci Sports. 2015;25:1-72. https://doi.org/10.1111/sms.12581.

34. Earhart GM, Falvo MJ. Parkinson disease and exercise. Compr Physiol. 2013; 3(2):833-48. https://doi.org/10.1002/cphy.c100047.

35. Hansen D, Dendale P, Coninx K, Vanhees L, Piepoli MF, Niebauer J, et al. The European Association of Preventive Cardiology Exercise Prescription in everyday practice and rehabilitative training (EXPERT) tool: a digital training and decision support system for optimized exercise prescription in cardiovascular disease. Concept, definitions and construction methodology. Eur J Prev Cardiol. 2017;24(10):1017-31. https://doi.org/10.1177/204748731 7702042.

36. Kennedy BK, Partridge L. 2nd interventions in aging conference. Aging (Albany NY). 2017;9(4):1090-5. https://doi.org/10.18632/aging.101221.

37. Eriksson M, Ng N. Changes in access to structural social capital and its influence on self-rated health over time for middle-aged men and women: a longitudinal study from northern Sweden. Soc Sci Med. 2015;130:250-8. https://doi.org/10.1016/j.socscimed.2015.02.029.

38. Sheng G, Xie Q, Wang R, Hu C, Zhong M, Zou Y. Waist-to-height ratio and non-alcoholic fatty liver disease in adults. BMC Gastroenterol. 2021;21(1):239. https://doi.org/10.1186/s12876-021-01824-3. 
39. Zou Y, Sheng G, Yu M, Xie G. The association between triglycerides and ectopic fat obesity: an inverted U-shaped curve. PLoS One. 2020;15(11): e0243068. https://doi.org/10.1371/journal.pone.0243068.

40. Palmer BF, Clegg DJ. The sexual dimorphism of obesity. Mol Cell Endocrinol. 2015:402:113-9. https://doi.org/10.1016/j.mce.2014.11.029.

41. Swainson MG, Batterham AM, Hind K. Age- and sex-specific reference intervals for visceral fat mass in adults. Int J Obes. 2020;44(2):289-96. https:// doi.org/10.1038/s41366-019-0393-1.

42. Kwon YM, Oh SW, Hwang SS, Lee C, Kwon H, Chung GE. Association of nonalcoholic fatty liver disease with components of metabolic syndrome according to body mass index in Korean adults. Am J Gastroenterol. 2012; 107(12):1852-8. https://doi.org/10.1038/ajg.2012.314.

43. Zou Y, Yu M, Sheng G. Association between fasting plasma glucose and nonalcoholic fatty liver disease in a nonobese Chinese population with normal blood lipid levels: a prospective cohort study. Lipids Health Dis. 2020;19(1):145. https://doi.org/10.1186/s12944-020-01326-3.

44. Black $\mathrm{N}$. Why we need observational studies to evaluate the effectiveness of health care. BMJ. 1996;312(7040):1215-8. https://doi.org/10.1136/bmj.312.704 0.1215 .

\section{Publisher's Note}

Springer Nature remains neutral with regard to jurisdictional claims in published maps and institutional affiliations.

Ready to submit your research? Choose BMC and benefit from:

- fast, convenient online submission

- thorough peer review by experienced researchers in your field

- rapid publication on acceptance

- support for research data, including large and complex data types

- gold Open Access which fosters wider collaboration and increased citations

- maximum visibility for your research: over $100 \mathrm{M}$ website views per year

At $\mathrm{BMC}$, research is always in progress.

Learn more biomedcentral.com/submissions 